\title{
CHIO DEI MAONESI SULLA FINE DEL TRECENTO (DAGLI ATTI DEL NOTAIO DONATO DI CHIAVARI)
}

Il notaio Donato di Chiavari lavorò a Genova negli anni 1374- 75. Nella primavera del 1389, probabilmente al seguito del podestà Antonio Leardo, partì per Pera, dove è documentata la sua attività dal $1^{\circ}$ ottobre 1389 al $1^{\circ}$ settembre 1390 . Successivamente troviamo il nostro notaio a Chio nel novembre del 1393. Rimase a Chio fino al 1408; poi ritornò in Liguria. Donato di Chiavari fu dunque uno di quei classici notai genovesi che, dopo un tirocinio in patria, svolsero buona parte della loro attività nelle sedi coloniali della Repubblica.

Gli atti pervenutici da lui rogati a Chio sono complessivamente centosei, per il periodo dal 17 febbraio al 12 novebre 1394, e si conservano nell'Archivio di Stato di Genova, nella filza n. 542. Pure non coprendo neppure l'intero corso dell'anno, questi documenti, pubblicati da Michel Balard, nel 1988, come volume 51 della Collana Storica di Fonti e Studi diretta da Geo Pistarino, presentano un interessante spaccato della vita economica di Chio in quel periodo, con alcuni riferimenti che risalgono addirittura al 1383 ed anni seguenti, grazie alle notizie inserte nei documenti sopracitati '.

Non mancano tra queste carte le notizie in base alle quali siamo in grado di ricostruire in questo momento la struttura della amministrazione chiota: podestà, vicario del podestà, gubernatores, massari, extimatores ecc. Nella nostra silloge sono numerosi gli atti di procura (si tenga presente la difficoltà di movimento connessa alla disponibilità dei trasporti via mare); si trovano contratti di cambio monetario, per cui, ad esempio, fiorini e gigliati di Chio vengono dati contro ducati oppure ducati d'oro vengono cambiati con lire genovesi o con bisanti bianchi di Famagosta $^{2}$; si hanno notizie di lettere di cambio '; compaiono esempi di contratti di affitto ${ }^{1}$ e di atti di cessione di immobili, come un viridarium situato nei borghi di Chio, in contracta Paricbie, o una casa sita nel castrum di Chio, in contrata Sant'Antonio, od altre case, site anch'esse nel castrum'. Vi sono atti di riconoscimento di

1 M. Balard, Notai Genovesi in Oltremare. Atti rogati a Cbio da Domato di Chiavari 17 febbraio- 12 novembre 1394). Collana storica di fonti e studi diretta da Geo Pistarino, 51, Genova, 1988.

2 Doxc. 6, 23, 92.

3 Docc. 47, 87, 92.

1 Dix. 98.

'Docc. 28, 42, 55, 100. 
debito ${ }^{6}$; di assicurazione '; di quietanza ${ }^{8}$; di prestito ${ }^{9}$, che talora rispondono alle esigenze dell'Officium provisionis grani civitatis Syi, i cui ufficiali sono congiuntamente latini (genovesi) e greci ${ }^{10}$; di perizie di immobili effettuate dagli extimatores del Comune di Chio (i quali dispongono di un proprio notaio), con conseguente giudizio del vicario del podestà ". C'è anche un contratto di famulato ${ }^{12}$.

La serie dei documenti pubblicati in extenso dal Balard si apre con un contratto di assicurazione della coca del cittadino genovese Pietro di Albaro, che è in procinto di partire da Chio per Genova, e si chiude con la quietanza con la quale Francesco de Campis del fu Giovanni riconosce di avere ricevuto da Bernardo Paterio, agente per conto del fratello Nicola, la somma di 400 ducati di cui Nicola gli era debitore.

Notiamo innanzi tutto la presenza in Chio non soltanto di immigrati genovesi e liguri, ma anche di persone provenienti da Famagosta, da Caffa, da Pera, cioè da altri insediamenti genovesi in Oriente. Tra gli estranei all'area genovese sono relativamente numerosi gli anconitani, alcuni dei quali sono residenti stabili, mentre altri appaiono in Chio unicamente in via temporanea per la conclusione di particolari negozi.

I burgenses chioti, attestati dagli atti del nostro notaio, sono almeno ventiquattro: tra essi troviamo oriundi di Padova, di Milano, di Parma, di Pontremoli. Recano invece semplicemente la qualifica di habitatores almeno diciotto individui, tra cuiuno di Negroponte. Tra burgenses e habitatores non mancano figure appartenenti a grosse famiglie genovesi e liguri: ad esempio, Andrea Vignoso, Antonio de Oppicis, Giovanni Grimaldi, Antonio Imperiale. Una famiglia notevolmente rappresentata in Chio è naturalmente quella dei Giustiniani, nei suoi vari rami, di cui il nostro notaio ricorda oltre una ventina di nomi. Tra coloro che recano la qualifica di habitatores non mancano i greci e gli ebrei. C'è anche -caso unico- un civis $S y i{ }^{13}$.

Si trova anche occasionalmente in Chio il frate Domenico de Alamania, precettore di Napoli e di Avignone, il quale è implicato nella pace conclusa tra il capitano ed il subasi di Smirne dopo l'arresto, da parte del capitano, di due figli del subasi ${ }^{14}$. Un altro straniero proveniente del Nord- Europa è Anechino di Bruges, che funge da serviente del podestà locale ".

6 Doc. 51.

Doc. 90.

Docc. 52, 58, 75, 87, 87a, 106.

9 Doc. 97.

10 Docc. 61, 96.

11 Docc. 63, 69, 70, 71.

12 Doc. 84.

13 Cfr. l'Indice dei nomi, alle voci Syi burgensis e Syi babitator, tenendo presente che fra i burgenses mancano i nomi di Bermardus Paterius, Pasqualinus de Pontremullo, Raffael de Coronato, Raffael Iosep, Simon Perellus de Vulturo, e fra gli babitatores manca il nome di Nicola Prasimo Nimicus, mentre devono essere eliminati i nomi di Pasqualinus de Pontremullo, Raffael de Coronato, Raffael losep, Sideri Canamicius. Il civis Syi è l'interprete Nicolò di Rapallo (doc. 56).

14 Docc. 77, 78, 79, 80.

15 Doc. 67. 
Sono presenti numerosi notai; ma non mancano anche gli esercenti dei consueti mestieri: da quello del banchiere a quello del balestriere, da quello del calegarius a quello del barberius, del cirurgicus, del medico o pbisicus. Circolano in Chio i ducati, i forini, i gigliati, i perperi.

I personaggi, che più di frequente compaiono nei nostri documenti e che quindi possono considerarsi, in un modo od in un altro ed a diverso livello, $i$ più attivi di questa società chiota, sono il dominus Bartolomeo Giustiniani di Francesco, i burgenses Andriolo de Costa del fu Antonio, Antonio de Summo, Bartolomeo de Guiso, Bernardo Paterio, Cristoforo de Costa, Donato de Via, Francesco de Campis del fu Giovanni, Giovanni di Coronata del fu Corrado, Giovanni Grimaldi, Ianoto di Milano, Manuele dl Parma, Nicola Paterio, Raffaele di Coronata, Raffaele Josep o Ioxepe, gli babitatores Antonio Imperiale e Antonio Sagimhem, ed inoltre Antonio Giustiniani de Roca o Rocha, Antonio de Rondanina, Battista Giustiniani del fu Lazzarino, Bernabò Dentuto, Cristoforo de Casali o Cesali del fu Raffo, Daniele Giustiniani olim Longus, il phisicus ebreo Eliseo Calaihi, Giorgio Giustiniani Arangio, Giovanni Giustiniani de Garibaldo, Giovanni de Luna, Marco de Bardi, Michele Lomellino, Raffaele Giustiniani olim Arangius del fu Francesco, Raimondo Bezacia, Tommaso di Bergamo. Come si vede, e come si è già detto, sono numerosi i membri della famiglia Giustiniani, dei quali abbiamo citato soltanto quelli che compaiono più di frequente, ma che, nel complesso, sono oltre una ventina e dominano la "piazza» ed i rapporti economici dell'isola.

Alcune altre figure emergono in modo particolare. Sono il burgensis Francesco Gardino o Goardino o Guardino, l'babitator Lazzaro o Lazzarino Nicola di Rapallo, il burgensis Pasqualino di Pontremoli, Simone Perello di Voltri. Francesco Gardino compare spesso come teste negli atti rogati dal nostro notaio, il che rivela una sua larga notorietà, derivatagli probabilmente dalla sua professione notarile e dal fatto che egli opera tra le varie componenti etniche dell' isola. Lo vediamo infatti nominato nel maggio del 1394 come procuratore dell'ebreo Ioil, abitante di Chio, come pure lo troviamo acquirente di una casa nel castrum chioto, contrada di Sant'Antonio, vendutagli il 5 giugno dal sacerdote greco Nicola Triandafilo di Crionia ${ }^{16}$. Roga un documento per Ottobuono Giustiniani il 9 febbraio $1393^{17}$, ed un altro per conto di Nicola di Portofino, padre ed amministratore di Gandolfo di Portofino, il 4 maggio del $1394^{18}$; è procuratore di Antonio Giustiniani de Rocha ${ }^{19}$, insieme al quale, il 26 gennaio 1394, ottiene dal locale podestà una sentenza favorevole contro Raimondo Bezacia ${ }^{20}$; è presente alla conferma del testamento del medesino Raimondo Bezacia il 24 giugno ${ }^{21}$. Francesco Gardino è in sostanza un notaio che fa parte della media attività della vita quotidiana; ma ci sembra significativa la frequenza con cui egli compare nella nostra documentazione.

16 Docc. 29, 42.

17 Doc. 104.

18 Doc. 63.

19 Docc. 70, 71

20 Doc. 82.

21 Doc. 53. Francesco Gardino compare come teste anche nei docc. 6, 7, 17, 33, 34, 36, 37, 68, 77, 81, 97 , 98. 
Ancora più attiva è la presenza di Lazzaro o Lazzarino Nicola di Rapallo, abitante di Chio. Ma questa sua presenza è limitata al fatto che egli compare soltanto come teste in ben ventisei atti sui centosei rogati da Donato di Chiavari ${ }^{22}$ : il che ripropone il problema della possibilità che vi fossero persone le quali si prestavano quasi sistematicamente a fungere da testimoni. Si noti infatti che i rogiti, nei quali il Nostro compare, sono nella grandissima maggioranza redatti ad staciam ubi ius redditur o iura reduntur per dominum potestatem Syi, oppure in aula palacii domini potestatis Syi. Solo quattro volte lo vediamo in altra sede. E' presumibile che rivestisse un ufficio od una occupazione nella curia podestarile, ma non sappiamo quale. Sappiamo però che conosceva la lingua greca, tant'è vero che in un documento funge da interprete ${ }^{23}$.

Pasqualino di Pontremoli è membro di una famiglia che comprende anche $\mathrm{i}$ suoi fratelli Francesco, Giacomo ed Antonio, anch'essi tutti qualificati come de Pontremulo, i quali sono immigrati dalla Lunigiana in Oriente. Egli è burgensis di Chio e risulta di frequente come testimone in atti rogati in sedi diverse. Non si tratta però e perciò di una situazione analoga a quella di Lazzaro o Lazzarino Nicola di Rapallo, perché Pasqualino figura anche direttamente implicato in questioni patrimoniali ed in contratti di affari. Il suo ci sembra il caso tipico di quei membri di numerose famiglie liguri che si ripartivano tra diversi stabilimenti coloniali onde meglio gestire una vasta rete di affari. Ed infatti il suo defunto fratello Francesco, burgense di Pera, lo ha lasciato fedecommissario dei suoi beni (ed egli nomina suoi procuratori i fratelli Giacomo ed Antonio ed il burgensis peroto Branca Spinola perché si occupino delle questioni relative alla successione) ${ }^{24}$; egli stesso ha ricevuto una somma cospicua di ducati di Chio in accomendacione da Dondedeo di Sant'Olcese, e nel giugno del 1394 paga il dovuto a Daniele Giustiniani olim Longus, che agisce in qualità di procuratore degli eredi di Dondedeo, il quale nel frattempo è defunto ${ }^{25}$. Nomina poi procuratore per tutti i suoi affari un certo Leonino Castagna, figlio di Tommaso ${ }^{26}$. Il fratello Antonio agisce quale teste in loco, accanto a lui, un paio di volte ${ }^{27}$. Pasqualino è un medio affarista, tipico esponente di quella cerchia di lunigianesi che sono venuti in colonia in cerca di fortuna.

Ancora diverso è il caso del notaio Simone Perello di Voltri. Lo troviamo mentre presta giuramento sulle Sacre Scritture il 18 febbraio 1394 quale vicinus di donna Agnese del fu Giacomo de Camolino di Candia, vedova di Bartolomeo de Caginaldo, abitante di Candia, in un atto di procura ${ }^{28}$. Poi, il 25 marzo, funge da curatore di Nicolosia del fu Raffo di Casale, vedova di Giovanni de Luna, ed in tale veste consegna alla medesima, per dote ed antefatto, una grande casa in Chio con

22 Docc. 6, 7, 9, 13, 17, 22, 29, 32, 33, 34, 42, 54, 55, 56, 57, 59, 62, 63, 72, 73, 81, 82, 84, 93, 95, 99.

23 Doc. 17.

24 Doc. 8.

25 Doc. 52

26 Doc. 54. Pasqualino compare come teste nei docc. 2, 26, 68, 78, 79, 80, 91, 92, 97, 98.

27 Docc. 91, 92.

28 Doc. 5. 
giardino, una seconda casa ancora in Chio, anelli, indumenti e denaro sonante ${ }^{29}$. Contestualmente Nicolosia cede al Perello, in favore delle figlie ed eredi del defunto marito, Eliana e Marieta, i propri diritti su altre tre case in Chio, appartenute al di lei fratello, Cristoforo di Casale, e riceve da lui tutti i diritti delle medesime sopra una bottega sita nel bazar chioto ${ }^{30}$. Simone agisce nuovamente come vicinus et consiliator in affari della sopracitata donna Agnese il 16 maggio; è interprete di ebraico e, grazie a ciò, si adopera per diversi ebrei dell'isola. Nel settembre del 1394 risulta avere sposato la sopraddetta Nicolosia del fu Raffo di Casale, vedova di Giovanni de Luna ${ }^{31}$. Quando funge da testimonio in atti notarili, questi rivestono importanza notevole o per il tipo di affare commerciale oppure perché vi sono implicati personaggi di alto rilievo, come nel caso degli accordi tra il subasi ed il capitano di Smirne il $I^{\circ}$ settembre 1394 . Simone è dunque persona di alta levatura culturale, apprezzato e richiesto nella migliore società chiota, certamente dotato di buoni mezzi economici.

L'intervento di Simone Perello come interprete in atti riguardanti gli ebrei di Chio pone in evidenza questo gruppo etnico-religioso, che comprende una ventina di individui, tra uomini e donne, $i$ quali non sono soggetti a restrizioni particolari, anzi appaiono inseriti con buone prospettive nella società locale. Hanno il loro rabbino; giurano super licteris ebraycis, more Iudeorum ${ }^{32}$; abitano in una loro contrada; esercitano nobili professioni, come quella del medico; effettuano grossi affari; fungono da testimoni in atti notarili fra $\mathrm{i}$ correligionari; usufruiscono in pieno della legislazione genovese, anche se non risultano investiti di cariche pubbliche ${ }^{33}$. In genere tuttavia operano nell'interno del proprio gruppo, sia pure servendosi dei notai latini; mostrano una spiccata propensione per le attività economiche; s'imparentano fra di loro e tra di loro determinano il circuito degli affari. Sono, comunque, buoni sudditi genovesi. Cosí quando Ihera Chimesti del fu Elia Comita, vedova di Elia Fapsoni, abitatrice di Chio, aggiunge un codicillo al proprio testamento, fatto il 25 dicembre 1393, per mano del notaio Donato di Chiavari, lega alla dominacio Syi una somma di denaro occasione aniotichi. Nel medesimo tempo, tuttavia, poiché il suo esecutore testamentario, Leonino, figlio di Beniamino Fochiano, ha rinunciato all' incarico, lo sostituisce con il rabbino Elias. L'atto è rogato nella casa di un'ebrea, Ihera Melicha, vedova di maestro Ismaele, ma i testi presenti sono tutti latini, anzi liguri, fatta eccezione per Manuele di Parma ${ }^{34}$.

Allo stesso modo il rabbino Elias ottiene senza particolari problemi che il vicario del podestà, Guglielmo di Ceva, faccia valutare dai pubblici extimatores del

29 Doc. 18.

30 Docc. 19, 20. 100.

31 Docc. 21, 31, 40, 81. Simone compare come teste nei docc. $21,40,41,55,70,71,78,80,97,98$,

32 Doc. 30

33 A meno che sia un ebreo il Raffaele Iosep o Ioxepe che risulta essere extimator del Comune di Chio insieme con Ianoto di Milano (docc. 22, 62, 63, 69, 70, 71).

34 Doc. 21. 
Comune di Chio alcuni beni immobili nel casale di Timiana, che vengono a lui assegnati in pagamento di un suo credito verso Nicola Tiliano ${ }^{3}$.

Non mancano comunque i rapporti tra ebrei e latini. Un caso sintomatico è quello di Joil, abitante di Chio, che nomina suo procuratore il notaio Francesco Gardino ${ }^{36}$. Più rilevante ancora è quello di Raffaele Catalano, giudeo, figlio di Sabata, il quale è in grossi rapporti di affari con il genovese Domenico Cattaneo, rappresentato da Giovanni Giustiniani de Garibaldo grazie ad un atto di procura fatto in Teologo per mano del figlio di detto Domenico, Isnardo, con le sottoscrizioni del console genovese in Teologo e di due testimoni: una procedura che, fra l'altro, ci rende edotti di come i genovesi procedessero per i propri atti giuridici là dove non avevano notai. Prestano cauzione altri sei ebrei per un totale di 350 ducati d'oro ${ }^{37}$.

C'è un gruppo di tre documenti che Donato di Chiavari roga nello stesso giorno, il 29 maggio, verso il vespro, uno dopo l'altro, esclusivamente per contraenti ebrei. Elias, figlio di Mosé Gripioti, e sua madre Ecsati, vedova di detto Mosé, vendono a maestro Eliseo Calaihi, phisicus, del fu maestro Mosé, una casa in Chio, nella contrada degli ebrei, per 225 ducati d'oro ${ }^{38}$. In questo caso è rilevante il fatto che, mentre due testimoni all' atto sono ebrei, gli altri due sono latini, anzi liguri. Lo stesso giorno maestro Eliseo concede ed Elias ed a sua madre, Ecsati, la facoltà di riscatto della casa entro il termine di quattro anni ${ }^{39}$. Anche questo documento, come il precedente, è rogato nella contrada dei giudei; ma ora i testimoni sono tutti latini, ed uno di essi, Simone Perello di Voltri, funge da interprete. Il terzo atto si svolge nella piazza locale, presso la loggia dei domini, avendo per testimoni esclusivamente due latini. Grazie ad esso il sopracitato maestro Eliseo riceve per conto di Melicha, figlia del fu Elias Salomon, vedova di maestro Ismail e tutrice del figlio Gedidia, tutto ciò che era pervenuto ad Aleramo Cibo, burgense di Rodi, sui beni di detto maestro Ismail ${ }^{40}$.

Ritroviamo maestro Eliseo, procuratore di Melicha, il 17 giugno, quando vende a Daniele Giustiniani olim Longus un quantitativo di mastice, prelevato sui contingenti di Damasco e di Alessandria e misurato al cantaro di Famagosta, che viene detto equivalente a sette centinaia e mezzo. I due acquirenti incaricano tre loro procuratori del ritiro del mastice " co Giustiniani de Campis la riscossione d'una somma di denaro dovuta dal defunto Nicola Giustiniani a maestro Ismail di Chio, fisicus, del fu maestro Callo ${ }^{42}$.

$\mathrm{Ma}$ Chio è anche un luogo di convergenza, dove cioè si incontrano e trattano affari giudei provenienti da sedi diverse. E' il caso di Samarias, nipote di maestro Barone, abitante a Pera, e di Iosep, figlio di Salomone, abitante a Candia, i quali, il

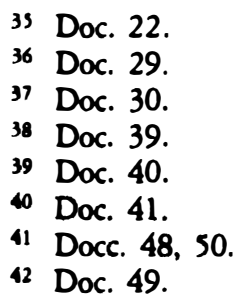


$I^{\circ}$ luglio 1394, nella stacia ubi ius redditur per dominum potestatem Syi, alla presenza dell'interprete Nicola di Rapallo e di due testi, certamente non ebrei, si rilasciano reciproca quietanza per tutti i loro affari ${ }^{43}$.

Non manca, tuttavia, l'ebreo che entra in comunione con latini e greci per finalità speculative. Maestro Eliseo, già a noi noto per altri affari con l'elemento latino di Chio, si unisce a Giovanni Giustiniani de Garibaldo, Donato de Via, Giorgio Diasormo, Bernardo Paterio e Dorino di Portofino per stipulare un contratto di assicurazione a favore dell' anconitano Petrello di Bonaggiunta per un trasporto di sapone, olio, carta, tappeti e canapa sulla coca di Bernabò Dentuto da Chio a Teo$\log 0^{44}$.

Infine un affare a tre, fra un ebreo, un greco ed un latino, stipulato il 28 aprile 1394, quando Elia Moscholia, giudeo, incarica il pope Leone di Costantinopoli di ritirare presso il notaio Guirardo Fontana una quietanza rilasciata in Pera da Elia Calaihi, giudeo ${ }^{45}$.

Rispetto agli Ebrei la presenza dei Greci tra i documenti di Donato di Chiavari appare ridotta. Forse i Greci esplicano una minore attività economica o piuttosto ricorrono meno di frequente all'opera del notaio? O forse usano sistemi di contrattazione per cui si servono di propri ufficiali? Oppure sono effettivamente minori -come però non crediamo- $\mathrm{i}$ loro rapporti contrattuali con i latini e gli occidentali in genere? Comunque, tre situazioni ci sembrano particolarmente significative. Si tratta sempre di rogiti tra contraenti greci e latini: non compaiono rogiti i cui contraenti siano soltanto greci.

Il primo caso riguarda il pope Nicola Triandafilo di Crionia, il quale vende al notaio Francesco Gardino, burgense di Chio, una casa nel castrum chiense, in contrada Sant'Antonio, con pagamento sul banco di Teodoro Sirichari, evidentemente anch'egli greco. Tra i cinque testimoni, due sono quasi sicuramente gre$\mathrm{Ci}^{46}$.

Il secondo rogito concerne la donazione con cui il greco Nicola Prasmo Nimico del fu Sidero Canamicii, abitante del borgo chioto, con il consenso della moglie, anch'essa greca, Cali Angelina del fu pope Giorgio Galatolla, cede a Cristoforo de Costa una casa nel borgo di Chio, in contracta Paricbie, con cortile e giardino, in ringraziamento dei servizi che Cristoforo gli ha reso in passato. La donna giura super maiestate, more Grecorum; compaiono fra i sei testimoni quattro greci: Teodoro Caloceto, Nicola Vlasto, Sidero Prasmo Canamicii e Sergi Vestarchi Galatolla, i quali sono rispettivamente il figlio ed il fratello di Angelina e le prestano assistenza come i parenti più prossimi ${ }^{47}$.

Il terzo rogito riguarda la procura che un greco, Manoli Iuncta del fu Nicoforo, abitante di Chio, rilascia ad un latino, Giovanni Giustiniani de Garibaldo, per la riscossione dei suoi crediti verso Ottobuono Giustiniani del fu Giovanni ${ }^{3}$. Interes-

\footnotetext{
43 Doc. 56.

44 Doc. 89.

45 Doc. 99

46 Doc. 42

47 Doc. 57.

48 Doc. 104
} 
sante rilevare come i greci dell'isola godessero di parità di azione verso i Giustiniani, i quali, come si sa, erano partecipi della Maona.

Come si svolge in Chio la vita di tutti i giorni? Ce lo dicono alcuni tipi di documenti: i contratti dotali, i testamenti ed i codicilli, le compravendite di schiavi.

Il documento con cui Nicola Franco, figlio di Giorgio Carvegni Franco, abitante di Chio, dichiara di avere ricevuto da Cristoforo de Costa, burgense chioto, la dote della propria moglie Marieta del fu Giovanni de Costa non presenta nulla di particolarmente interessante, salvo il fatto che vi assiste un interprete: il che significa che gl'interessati sono alloglotti. Notiamo che Cristoforo de Costa non èl padre della sposa, ma agisce come se fosse tale e fruisce illis privilegiis et gratiis quibus pater gaudeat pro filia sua, tant'è vero che Marieta deve impegnarsi a non redigere, vivente Cristoforo, nessun testamento, codicillo, contratto, obbligazione ${ }^{49}$.

Un membro d'una importante famiglia di Pera, Iane Demerode del fu Filippo, burgense di Pera, sposa una donna d'una altrettanto importante famiglia di Chio: Perpetua, figlia di Francesco Giustiniani de Campis. Avvenuta la transductio in domum viri, il 30 luglio 1394 il padre della sposa paga al marito la dote stabilita, 800 lire di Genova, mentre il marito costituisce un antefactum di pari ammontare. Ma forse proprio perché siamo tra gente di alto livello economico, c'è una grossa questione finanziaria a proposito della dote. Iane Demerode asserisce che, secondo i patti nuziali, il suocero avrebbe dovuto versargli, oltre alle 800 lire di dote, anche 400 lire pro guarnimento, cioè per il corredo della sposa. Bartolomeo, altro figlio di Francesco e fratello di Perpetua, ribatte che il padre aveva promesso al futuro genero soltanto 800 lire pro dotibus et patrimonio della donna. Si viene ad un accordo: Iane rinuncia ad ogni pretesa e diritto sulle 400 lire per il corredo della sposa; Francesco rinuncia a richiedere un nuovo antefactum allo sposo qualora si decida a corrispondere a Iane le 400 lire contestate; Iane vende, o, meglio, dona a Bartolomeo un "luogo" della Compera Venetorum per il valore di 90 lire di Genova ${ }^{\text {so }}$.

Se le costituzioni dotali presentano in Chio problemi che possono definirsi inusuali in Genova, una analoga varietà di situazioni si presenta per i testamenti, dovuta appunto alle specifiche circostanze ambientali dell'isola greco-genovese. Siamo nella norma consueta con un inventario di beni del 19 febbraio 1394, lasciati da Bartolomeo Vignoso e di cui sono responsabili Andrea Vignoso, burgense di Chio, e Pietro di Albaro. E' un inventario relativamente ricco, che comprende tre tazze e dieci cucchiai d'argento, un guanciale e quattro vellate, una borsa di cremisi ricamata, sette tovaglioli ricamati d'oro e di seta, un tovagliolo ricamato dall'orlo vermiglio, una tunica foderata di volpe, un giubbone doppio di panno bianco foderato di pelle di lupo ". Rimaniamo ugualmente nella norma con il documento del 21 febbraio 1394 con cui Pasqualino di Pontremoli, fedecommissario del defunto fratello Francesco, burgense di Pera, non potendo recarsi da Chio a Pera per

\footnotetext{
49 Doc. 17.

so Docc. 64, 65, 66.

31 Doc. 7.
} 
eseguire le volontà dello scomparso, nomina dei procuratori ad boc "22. Abbiamo già accennato al codicillo che l'ebrea Ihera Chimesti aggiunge il 5 aprile 1394 al proprio testamento del precedente 25 dicembre, con un lascito a favore della Maona di Chio ". Annotiamo che per la sostituzione di un curatore testamentario, che affida ad un terzo la procura per una eredità giacente (come nel caso di quella del defunto Clariel de Corvo di Monleone), occorre una sentenza del vicario del podestà e governatore di Chio ${ }^{34}$.

Ma il caso più interessante, perché specifico della presenza genovese nel mondo greco e che trova un parallelo in un fatto quasi analogo verificatosi a Pera nel 1386 ", è quello di Raimondo Bezacia del fu Giacomo: un maonese che traffica con Pera, possiede beni immobili in Chio e la castellania del castrum Amistai, ed è aiutato nei propri affari dal figlio Raffaele, che traffica a sua volta con Famagosta ${ }^{56}$. Il 22 giugno 1394 Raimondo detta e sottoscrive il proprio testamento all'uso romanobizantino, cioè con la firma ed i sigilli di sette testimoni: Guirardo Pancia, Bartolomeo de Guiso, Antonio Imperiale, Ianoto di Milano, Iane Argenti, Giorgio de Nigro, Raffo Specia. Ma non basta. Non sappiamo per quale motivo il Bezacia sia ricorso a questa procedura. Vediamo però che due giorni dopo, in contracta ecclesie episcopalis, Raimondo conferma, per mano di notaio ed alla presenza di tre testimoni, che quello è il suo reale ed ultimo testamento ". Non si è certi, insomma, della validità della procedura romano-bizantina e si vogliono quindi evitare le eventuali contestazioni o ricorrendo alla convalida del vicario del podestà, come nel caso di Pera, o rifacendosi in qualche modo all'uso notarile occidentale.

Non mancano pertanto -e sono $\mathrm{i}$ casi più frequenti- $\mathrm{i}$ testamenti redatti per mano notarile. Abbiamo due esempi, di due grossi personaggi. Uno è Pietro Giustiniani olim de Rocha del fu Simone: se morirà al di fuori di Genova, vuole essere sepolto in una ex ecclexiis Cbristianorum, secundum locum in quo decedet; lascia ai poveri, sino all' ammontare di 200 lire, il ricavato della vendita dei suoi vestiti e degli axnixia apta ad navigandum; lega alla figlia naturale, Caterina, 300 lire quale dote per il matrimonio, prima del quale ella sarà mantenuta a carico dell'asse ereditario (in caso di contestazione, le 300 lire passeranno come legato a Battista Giustiniani del fu Lazzarino, che ne disporrà secondo la volontà espressagli dal testatore). Saranno sue eredi la madre Luchina Giustiniani e la cognata Pietrina, vedova di suo fratello Simone, oppure la sola Luchina, se Pietrina si risposerà e non continuerà a vivere con i figli.

Pietro Giustiniani dispone in Pera di una racio di 4.600 perperi, in cui sono compartecipi Ianoto Bezacia ed Antonio Giustiniani de Rocha; possiede inoltre i

32 Doc. 8.

33 Doc. 21

s4 Docc. 33, 34.

35 L. BalletTo, Pera genovese negli atti del notaio Donato di Cbiavari (1389-90), relazione al IV Symposium «Bulgaria Pontica Medii Aevin (Nessebar - Bulgaria, 26-30 maggio 1988), pubblicato in * Atti dell' Accademia ligure di scienze e letteren, XLVI (1989), Genova, 1990, pp. 457-472. Per i regesti degli atti peroti di Donato di Chiavari cfr. M.BALARD, Péra an XIVe siècle. Documents notariés des Archives de Gênes, in M.BALARDA.E.LAIOU-C.OTTEN-FrOUX, Les Italiens à Byzance, Paris, 1987, pp. 30-50.

36 Docc. 35, 45, 46, 51, 57, 70, 71 .

37 Doc. 53. 
beni, le merci ed i denari pervenutigli dal suo defunto fratello Simone. Se egli morirà in partibus orientalibus, i suoi beni ed il suo cartulario passeranno al suddetto Ianoto Bezacia, burgense di Pera, che provvederà a crediti e debiti, impegnando i proventi in locis comunis Peyre vel aliis locis a suo giudizio, finché nova babuerit civitatem Ianue pacificari ed in pacifico vivere ${ }^{98}$. Anche a Chio o nelle parti orientali il richiamo alla patria genovese non si dimentica. Genova attraversa un momento difficile per gl'interni contrasti che la porteranno in dominio della Francia, ed a Chio si ripercuotono i riflessi della situazione.

Un altro testamento riguarda un fiorentino, ed è proprio questo fatto a renderlo interessante. Emergono infatti di qui non soltanto i rapporti che il testatore mantiene con Firenze, ma anche la rete dei traffici da lui intessuti con Rodi e con Teologo, sulla costa asiatica, e degli affari stipulati con i Genovesi. Sono balle e fardelli di panni, tra cui panni inglesi e scaperoni, da portare a Rodi, sulla coca di Bernabò Dentuto; altri panni inglesi ed un fardello di arnesi da caricare sulla medesima coca a Teologo (dove agisce per il testatore il genovese Bartolomeo de Castro) e da portare ugualmente a Rodi; una grossa cassa piena di vesti e panni vecchi caricata a Chio, sempre sulla medesima coca, ed ancora con destinazione Rodi. Tutti questi beni devono essere consegnati in Rodi a Maurizio Ceba e Ceprano de Albertis (che agiscono nell' isola per il testatore), i quali ne disporranno secondo la volontà di Alamanno e Lodisio Manimi e loro fratelli di Firenze.

Il nostro fiorentino possiede in Chio una cassa con 120 ducati d'oro di Venezia; è creditore nei confronti di Ambrogio de Mari di 30 fiorini, concessigli in mutuo e che saranno pagati in Rodi ai sopracitati Maurizio Ceba e Ceprano de Albertis; è debitore verso Lorenzo Cibo, che si trova in Teologo, di 5 fiorini o pressappoco, e verso Ottaviano Imperiale di 1 fiorino d'oro. Lascia eredi dei suoi beni i propri fratelli Giacomo e Bertino; designa esecutore delle sue ultime volontà Giustiniano Giustiniani, figlio di Pietro, al quale competerà di disporre, alla sua morte, dei beni, arnesi e merci del testatore e di una sua schiava. E' interessante notare che i sette testimoni all'atto sono tutti liguri, fatta eccezione per un solo fiorentino. Veri Francisci Gori è dunque un tipico esempio della capacità di integrazione dei fiorentini e dei forestieri in genere nella società genovese di Chio, tant'è vero che egli vuole essere sepolto nella locale chiesa di Sant'Antonio".

Sono poi relativamente frequenti nella nostra silloge gli atti conseguenti alle disposizioni testamentarie. Cito ad esempio il rogito con cui il 26 ottobre 1394 frate Giovanni eremita e Giovanni Giustiniani de Garibaldo, esecutori testamentari del fu Pietro de Laurenciis, dichiarano di avere ricevuto da un debitore del defunto, certo Bernardo de Sesegna, burgense di Chio, la somma di 130 fiorini, 3 gigliati e 3 denari di Chio, quale residuo dell' importo di 296 fiorini, 1 gigliato e 14 denari, dovuto da Bernardo e pervenutogli dai beni di Pietro ${ }^{60}$.

Abbiamo tenuto per ultima la questione del testamento di Pisano Pelopoli di Ancona perché essa prospetta una situazione di rapporti giuridici internazionali e

38 Doc. 76.

39 Doc. 105.

60 Doc. 103. 
comprova la validità di certe procedure anche al di fuori della giurisdizione genovese. Pisano ha fatto testamento nel 1393 in Chio per mano del notaio Donato di Chiavari, nominando eredi le figlie Francesca e Margarella, sotto la tutela della madre Girella Zineta. La tutela è stata confermata dal podestà di Ancona, per mano di notaio anconitano, il 26 giugno 1394. Pietro Lombarducii e Giacomo Symoni di Ancona sono stati nominati agenti di Girella, con strumento dello stesso notaio anconitano, il successivo 21 agosto. Il 25 settembre, in Chio, essi dichiarano di avere ricevuto il ricavato di una partita di legname, depositato da Pisano presso Nicola di Santo Stefano in Chio, ed una ricca serie di oggetti e denari appartenuti a Pisano ${ }^{61}$. Non c'è qui soltanto una testimonianza di rapporti tra Chio ed Ancona; c'è anche la riprova della validità di atti notarili anconitani in Chio e viceversa, quindi della funzione internazionale del notariato.

Un aspetto significativo della vita sociale chiense è rappresentato dalla presenza e dalla compravendita degli schiavi. Ci sono rimasti in proposito alcuni documenti, $\mathrm{i}$ quali ci servono per esaminare il problema sotto diversi aspetti.

Il 28 agosto 1394 Bartolomeo di Padova, burgense chioto, vende a Pietro Giustiniani olim de Rocha lo schiavo tartaro Giorgio, dell'età di circa venti anni, per il prezzo di 46 ducati d'oro. Il 19 settembre frate Pace di Gubbio sporge querela di fronte al podestà di Chio contro Raffo Specia, cittadino di Genova, che gli ha venduto lo schiavo Martino, dell'età di circa diciotto anni, dichiarandolo sano e privo di difetti occulti. Invece frate Pace ha scoperto che lo schiavo ha una tibia più grossa dell'altra, sicché non può essere utile come dovrebbe. Inoltres urinam tenere non potest nec vallet, et maxime dormiendo, quia urinas non sentit urinare, que est magagna occulta. Egli perciò ha chiesto ripetutamente al venditore di riprendersi lo schiavo e di restituirgli il prezzo convenuto; ma Raffo non se ne è dato per inteso. Anche ora, di fronte al podestà, nicbil respondit nec disit ${ }^{62}$.

Il 24 ottobre Giovanni di Coronata, burgense di Chio, procuratore di donna Iacha, vedova di Nicola Macalufo, e della propria moglie Lucia, figlia di detto Nicola, vende a Iane Capadoca di Candia, per il prezzo di 40 ducati d'oro, lo schiavo circasso Andrea, dell'età di circa diciotto anni, sano e privo di difetti ${ }^{63}$.

Sono dunque presenti nell'isola schiavi orientali provenienti dall'area del Mar Nero e in prevalenza (almeno a quanto si desume dai nostri documenti) di sesso maschile. Il loro prezzo è notevole. E' interessante rilevare che per la vendita dello schiavo tartaro Giorgio e dello schiavo circasso Andrea si sottolineano la presenza ed il consenso degl'interessati: il che in sostanza attribuisce una certa personalità giuridica ed una certa facoltà di azione a questi schiavi medesimi. E ciò può significare una loro sorta di integrazione nella società di cui fanno parte. Il caso dello schiavo Martino è sintomatico di situazioni che dovevano essere forse più frequenti di quanto risulti documentato; cioè il tentativo di frode da parte dei venditori che non denunciano i difetti della merce, sì che l'acquirente si vede infine costretto a

61 Doc. 93.

62 Docc. 74, 86.

63 Doc. 102 
rivolgersi alla giustizia per ottenere soddisfazione del danno subito. Vizi e difetti fisici di questi mancipia non erano cosa rara, soprattutto per l'incontinenza orinaria, mentre un caso particolare è la probabile elefantiasi di cui Martino soffre ad un arto o la conseguenza di una ferita riportata alla tibia.

Un caso particolarmente interessante perché piuttosto raro e per il suo valore di carattere generale è quello di Micheleto di Perugia, veneto della Canea, che ha comperato dal sensale Antonio Coca, burgense di Pera, agente a nome di Antonio Bussoroto, uno schiavo tartaro dell' età di ventiquattro anni, e ciò con strumento notarile redatto in Pera il 18 maggio 1394. Ma lo schiavo si è presentato di fronte al podestà di Chio dichiarando di essere cristiano cattolico, di non essere mai stato schiavo, di essere quindi uomo libero di pieno diritto. Visti i testimoni da lui presentati, il podestà dichiara libero lo schiavo, mentre Micheleto reclama da Antonio Coca la restituzione del prezzo di 15 ducati d'oro, come riconosciutogli dal podestà. Andreoto di Lerici, burgense di Caffa, agendo a nome di Antonio Coca, paga la somma richiesta, e Micheleto cede ad esso Andreoto tutti i suoi diritti contro Antonio Coca ed Antonio Bussoroto ${ }^{64}$. Abbiamo dunque un esempio concreto della dichiarazione di libertà di uno schiavo quando questi si dice di religione cattolica, convalidando la dichiarazione con la presenza di testimoni. Resta però il fatto che il danno ricade sul venditore, a quanto pare anche se egli è in buona fede e soprattutto se non sa o non ha la possibilità di rivalersi su altri. $\mathrm{Ci}$ chiediamo comunque se questo procedimento fosse riserbato soltanto ai cristiani cattolici o se invece ai estendesse ai cristiani ortodossi.

Chio è un grande centro di commerci ed un porto frequentato da navi di varia specie in arrivo ed in partenza. La coca «Santa Maria» del genovese Pietro di Albaro si prepara a partire per Genova nel febbraio del $1394^{\circ \text {. }}$. Il successivo 15 aprile Bernardo di Recco, burgense di Rodi, dominus et patronus di una coca castigliana, la noleggia al dominus Antonio Giustiniani de Rocha per un viaggio alle isole di Nasso o Paro e quindi a Milo per effettuarvi un carico di grano ed orzo da trasportare a Rodi ed eventualmente di qui a Tripoli di Siria ${ }^{66}$. Manuele Doria ha concesso a nolo la propria coca castigliana a Francesco de Medio, veneziano di Candia, per un viaggio da Focea Vecchia a Mitilene per caricarvi bestiame, con ritorno a Focea Nuova per completare il carico di 50 bestie e di una quantità di mole da mulino, e quindi per il trasporto al porto di Candia. Sennonché Manuele, effettuato il carico, si è recato ed è rimasto fermo a Chio: donde una controversia giudiziaria, in cui Manuele dichiara di avere effettuato la diversione onde sbarcare a Chio il proprio fratello e si dice pronto a riprendere il viaggio ${ }^{67}$.

Un caso analogo è quello di Andalò de Mari, che ha stipulato un contratto di noleggio della sua coca "Corpus Christi» con il fratello Pietro de Mari per un viaggio

\footnotetext{
64 Doc. 43.

63 Docc. 1, 4, 6.

66 Doc. 26.

67 Doc. 59.
} 
diretto da Valenza a Barcellona, Maiorca ed Alessandria, ed invece ha fatto scalo a Chio. Questa volta la giustificazione è data da un attacco di pirati in prossimità dell' isola di Creta, i quali hanno catturato dodici uomini dell'equipaggio e si sono impadroniti di certi materiali: donde la necessità, per rimediare alla perdita, dello scalo a Chio, dove però Andalò non ha trovato il necessario né gli uomini disposti all'imbarco. Comunque, se Pietro lo desidera, Andalò si dice pronto a continuare il viaggio con gli uomini ed $i$ fornimenti di cui dispone ${ }^{68}$.

Il pericolo dei pirati (specificamente in questo caso dei pirati catalani) incombe anche a proposito del contratto che Gregorio Cicala, cittadino genovese, patrono della coca "San Giovanni Battista», ha concluso con nove mercanti, in maggioranza genovesi, e con un anconitano per un viaggio da Pera a Chio, Teologo, Rodi e Famagosta. La presenza, nei pressi di Rodi, di due navi pirate catalane, che già hanno catturato la coca di Benedetto Cibo, induce ad un nuovo contratto di noleggio, stipulato in Chio il 22 agosto, per la rotta diretta a Famagosta, senza le soste a Teologo e Rodi ${ }^{69}$.

Anche Bernabò Dentuto è proprietario e patrono di una coca che naviga in lungo ed in largo tra il Mediterraneo occidentale e l'Egeo. Ha trasportato e scaricato a Chio due balle di panni di Bruges, di proprietà di Battista Lomellino, caricate a Bruges e destinate a Gaeta per sua dichiarazione, a Siviglia per dichiarazione di Daniele Giustiniani Longo, agente di Napoleone Lomellino e figli, e non accettate né a Gaeta né a Siviglia: donde la consegna a Chio nelle mani di Daniele Giustiniani ${ }^{70}$. A Chio Bernabò s'impegna con Petrello di Bonaggiunta di Ancona per un trasporto a Teologo di un carico di sapone, olio, carta, tappeti, canapa ed altre merci, assicurato per 2.200 ducati $^{n}$.

Alcuni dati generali emergono da questa breve rassegna di navi e di trasporti. $\mathrm{Da}$ Chio si traffica con Genova, la Spagna, l'Italia meridionale, Alessandria, Cipro, Tripoli di Siria, Teologo, Creta, Rodi e le isole dell'Egeo, Pera... Le navi sono le grosse coche da trasporto, talvolta nella specifica tipologia delle castigliane. Un pericolo ricorrente è costituito dai pirati, che costringono talora le navi a modificare la rotta. Non tutti i carichi fanno capo a Chio, in arrivo od in partenza; talvolta queste navi chiensi operano esclusivamente tra località esterne. Proprietari e patroni sono genovesi; ma tra i clienti, in maggioranza genovesi, non mancano persone di altra provenienza: ad esempio, anconitani e veneziani. Le merci che queste navi trasportano sono le più varie: delle vettovaglie e dal bestiame alle mole da macina, alle stoffe, alla carta.

Certo nell'isola la maggior parte degli affari si esplicano per via di mare. Si tenga presente che il vettovagliamento è un fatto fondamentale, sì che esiste in Chio un Officium provisionis grani ${ }^{12}$. Non si tratta soltanto del grano, pure essendo questo il primo genere di vita. C'è anche, in primo piano, la questione dell'olio. Può essere un fatto di commercio interno quello delle 250 botti di olio vendute da Filippo

\footnotetext{
68 Doc. 60.

69 Doc. 73.

70 Doc. 88.

71 Docc. 89, 90.
} 
Scoto, civis et mercator genovese, a Francesco Giustiniani ${ }^{13}$. Sappiamo però che l'olio era anche oggetto d'importazione nell'isola. E' sintomatico il caso di Ansaldo Lomellino, che ha importato nell' isola una certa quantità di olio, sulla quale l'emptor et collector introytus olei ha imposto il pagamento di una tassa d'ingresso, mentre, secondo le convenzioni stipulate tra il Comune di Genova e la Maona, i genovesi godono dell'esenzione da dazio. Il percettore delle tasse, Antonio Sagimbem, di fronte alle proteste del Lomellino, risponde, dinanzi al vicario del podestà, che, secondo il contratto di vendita dell' introytus, fattogli da parte della Maona tramite il podestà ed i gubernatores, nessuno risulta godere di esenzione; inoltre richiede che $\mathrm{i}$ gubernatores e massari intervengano a sua difesa ${ }^{74}$. E' chiaro che manca la sintonia tra gli accordi stipulati dalla Maona con il Comune genovese e l'applicazione di quegli accordi in merito alle tasse doganali, anche se rimane a noi la curiosità di sapere se tale situazione fosse generica o specifica e se si limitasse all'importazione dell'olio o riguardasse qualunque genere di merci. Certo provengono dal commercio marittimo anche le tredici pezze di panno di Vervicq che Daniele Giustiniani olim Longus vende ad Antonio Imperiale del fu Adriano, a Criti Coresi ed a Giorgio di Negroponte", come pure i panni di Valeriano Lomellino venduti tramite Michele Lomellino ad Antonio de Rondanina, cittadino di Genova ${ }^{76}$. Quando però lo stesso Michele Lomellino vende una pezza di camocato a Manuele di Parma, burgense di Chio, il quale agisce anche per conto del balestriere Guirardo Schalia ", siamo al commercio capillare di queste mercanzie d'importazione.

Rimaniamo al traffico interno con i cuoi di becco de tribus sortis che i macellai Teodoro Mondano e Nicola Mangosi vendono al maestro Pietro di Vernazza ${ }^{78}$, mentre entriamo nel settore dell'esportazione in grande stile con il contratto di vendita di grossi quantitativi di mastice destinati alla Siria, all'Egitto, alla Romania ed alla Turchia, addirittura per otto e dieci anni ${ }^{79}$, oppure (ma forse per movimento interno?) per il mastice che maestro Eliseo, già citato, procuratore di Melica del fu Elias Salomon, vedova di maestro Ismail, vende a Daniele Giustiniani olim Longus ed a Bartolomeo Giustiniani olim de Campis, prelevandolo dai contingenti di Alessandria e-Damasco: e ciò con un contratto in cui maestro Eliseo s'impegna giurando super licteris ebraicis more Iudeorum ${ }^{80}$.

Un altro grosso commercio è quello dell'allume di Focea, per il quale i nostri documenti ci presentano il caso specifico e sintomatico d'una questione insorta fra $i$ governatori della Maona del 1393 e gli appaltatori di Focea Nuova in merito ai diritti fiscali di estrazione e di esportazione. In tale circostanza veniamo a sapere che l'allume di Focea interessava anche acquirenti turchi e che Focea era luogo di rifugio

72 Docc. 61, 96.

73 Doc. 15.

74 Docc. 9, 13, 14.

75 Doc. 24. I panni appartengono a Battista Lomellino.

76 Doc. 25.

77 Doc. 38.

78 Doc. 44.

79 Docc. 45, 46.

80 Docc. 48, 50. 
di schiavi fuggiti dalla Turchia: il che complicava le cose per il governo locale. Né mancano riferimenti interessanti circa le modalità ed il funzionamento degli appalti delle allumiere, soprattutto circa l'intervento del podestà di Chio ${ }^{81}$.

Compare infine tra i nostri documenti il movimento del denaro: si veda il caso dei 1419 bisanti che Battista Gattilusio consegna in Famagosta, tramite Giuliano Macaluffo, a Giovanni di Coronata, agente a nome di Iacha, vedova di Nicola Macaluffo, ed a nome di Lucia, figlia del medesimo e sposa di Giovanni, eredi testamentarie di Nicola, i cui capitali, evidentemente, erano anche impegnati in $\mathrm{Ci}$ pro $^{82}$.

Dunque, nel complesso: un'attività economica sia nel traffico di export-import, sia nelle dimensioni locali, in una grande varietà di prodotti, tra i quali naturalmente primeggia, per l'esportazione, il mastice. I grandi come i piccoli operatori di questi traffici sono innanzi tutto genovesi ed accanto a loro, ma -almeno in apparenza- in misura minore, i greci isolani. Non vi sono comunque discriminazioni: partecipano in parità gli altri occidentali, a partire dai veneziani e dagli anconitani, mentre sono ben attivi gli ebrei. Chio è, sotto molteplici aspetti, un punto di convergenza, tale da potersi definire anch'essa non soltanto «un'altra Genova», ma addirittura la capitale dell'Egeo genovese ${ }^{83}$.

\section{LAURA BALLETTO \\ Instituto di Medievistica Università di Genova}

81 Docc. 2, 10, 11

82 Doc. 91.

83 Per la bibliografia su Chio cfr. fra l'altro (ed ivi la bibliografia citata): D. Promis, La zecca di Scio durante il dominio dei Genovesi, in «Memorie della Reale Accademia delle Scienze di Torinon, II serie, XXIII, «Scienze morali, storiche e filologichen, Torino, 1866, pp. 325-383; C. Hopf, Storia dei Giustiniani di Genova, traduz. italiana di A. Wolf, in «Giornale ligustico di archeologia, storia e belle arti», anno VII e VIII, 1881, pp. 316-330, 362-373, 400-409, 471-477, anno IX, 1882, PP. 13-28, 49-65, 100-130; G. HEYD, Storia del commercio del Levante nel medioevo, traduz. italiana, Torino, 1913, passim; W. MILlER, The Zaccaria of Phocaea and Chios (1275-1329), in W. Miller, Essays on the Latin Orient, Cambridge, 1921 , ristampa anastatica Amsterdam, 1964, pp. 283-300; Id., The Genoese in Chios (1346-1566), ibidem, PP. 303-313; R.S. LÓPEZ, Genova marinara nel Duecento. Benedetto Zaccaria ammiraglio e mercante, Milano-Messina, 1933, passim; H. Giustiniani, History of Chios, ediz. a cura di PH. P ARGenti, Cambridge, 1943; L. GATto, Per la storia di Martino Zaccaria signore di Chio, in «Bullettino dell'Archivio Paleografico Italiano, n. s. II-III, 1956-1957, parte I, Pp. 325-345; PH. P. ARGENTI, The occupation of Chios by the Genoese and their administration of the island (1346- 1566), vol. I, Cambridge, 1958; G. PISTARINO, Nella "Romania" genovese tra i Greci e $i$ Turchi: l'isola di Chio, in «Rivista storica italiana", LXXIII. 1, 1961, pp. 69-84; Id., Chio dei Genovesi, in «Studi medievalin, 3." serie, X.1, 1969, pp. 3-68; M. BALARD, La Romanie Génoise (Xlle-debut du XVe siècle), Genova-Roma, 1978, passim; L. BALletTo, Chio nel tempo di Cristoforo Colombo, in *Atti del III Convegno internazionale di studi colombiani, Genova, 7 e 8 ottobre 1977n, Genova 1979, pp. 175-198; S. OrIGONE, Chio nel tempo della caduta di Costantinopoli, in aSaggi e documenti II", tomo I, Civico Istituto Colombiano, Studi e testi - Serie storica a cura di Geo Pistarino, 3, Genova, 1982, pp. 121-224; G.PISTARJNo, duecento cinquant'anni dei Genovesi a Chio, in G.Pistarino, Genovesi d'Oriente, Civico Istituto Colombiano, Fonti e testi Serie storica, 12, Genova, 1990, pp. 243-280.

Sullo specifico problema degli ebrei a Chio cfr. D. JACOBY, The Jews in Cbios under Genoese rule (1346-1566), in «Zion. A quarterly for research in Jewish history", XxVI, 1961, Pp. 180-197; P. VILLA, Documenti sugli ebrei a Chio nel 1394, in «Atti della Società ligure di storia patria», n. s. V.1, 1965, PP. 117-151; PH. P. ARGENTI, The religious minorities of Chios. Jews and Roman Catholics, Cambridge, 1970; G.G. Musso, Gli Ebrei nel Levante genovese: ricerche d'archivio, in «La Berion, X.2, maggio-agosto 1970, Pp. 5-27; M. BALARD, La Romanie Génoise cit., Pp. 279, 335337. 